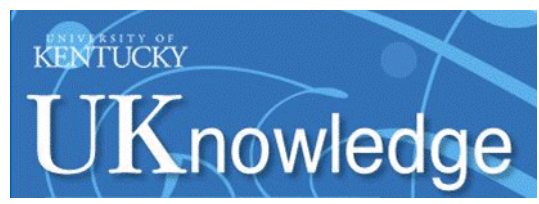

University of Kentucky

UKnowledge

$10-20-2000$

\title{
Observational Constraints on the Internal Velocity Field of Quasar Emission-Line Clouds
}

\author{
Mark Bottorff \\ University of Kentucky \\ Gary J. Ferland \\ University of Kentucky, gary@uky.edu \\ Jack Baldwin \\ Michigan State University \\ Kirk Korista \\ Western Michigan University
}

Follow this and additional works at: https://uknowledge.uky.edu/physastron_facpub

Part of the Astrophysics and Astronomy Commons, and the Physics Commons

Right click to open a feedback form in a new tab to let us know how this document benefits you.

\section{Repository Citation}

Bottorff, Mark; Ferland, Gary J.; Baldwin, Jack; and Korista, Kirk, "Observational Constraints on the Internal Velocity Field of Quasar Emission-Line Clouds" (2000). Physics and Astronomy Faculty Publications. 109. https://uknowledge.uky.edu/physastron_facpub/109

This Article is brought to you for free and open access by the Physics and Astronomy at UKnowledge. It has been accepted for inclusion in Physics and Astronomy Faculty Publications by an authorized administrator of UKnowledge. For more information, please contact UKnowledge@lsv.uky.edu. 


\title{
Observational Constraints on the Internal Velocity Field of Quasar Emission-Line Clouds
}

\author{
Digital Object Identifier (DOI) \\ http://dx.doi.org/10.1086/317051 \\ Notes/Citation Information \\ Published in The Astrophysical Journal, v. 542, no. 2, p. 644-654. \\ (C) 2000. The American Astronomical Society. All rights reserved. Printed in the U.S.A. \\ The copyright holder has granted permission for posting the article here.
}

This article is available at UKnowledge: https://uknowledge.uky.edu/physastron_facpub/109 


\title{
OBSERVATIONAL CONSTRAINTS ON THE INTERNAL VELOCITY FIELD OF QUASAR EMISSION-LINE CLOUDS
}

\author{
MARK BOTTORFF AND GARY FERLAND \\ Physics Department, University of Kentucky, Lexington, KY 40506 \\ JACK BALDWIN \\ Department of Physics and Astronomy, Michigan State University, East Lansing, MI 48824-1116 \\ AND \\ KIRK KORISTA \\ Physics Department, Western Michigan University, Kalamazoo, MI 49008 \\ Received 2000 March 8; accepted 2000 June 7
}

\begin{abstract}
This paper addresses the question, what does the spectrum of a typical quasar reveal about the velocity structure within its broad emission line region clouds? Turbulent (i.e., nonthermal) broadening of spectral lines can be due to macroturbulence or microturbulence. Microturbulence affects line formation and the emitted spectrum and may be required to account for the observed smoothness of the line profiles. The velocity field is crucial since it addresses the fundamental nature of the individual clouds and the global structure of the active galactic nuclei (AGNs) environment. For example, stellar winds or magnetically confined blobs might be highly microturbulent, requiring only a few internally broadened clouds to account for the observed smooth line profiles in AGNs. On the other hand, clouds in pressure confinement would have only thermal line widths, requiring many clouds moving in a large-scale velocity field to achieve the same effect. There are almost no previous studies of the effects of microturbulence, even though the observation that AGN lines are very smooth seems to require additional line broadening mechanisms. We present a broad range of photoionization calculations in which the microturbulence is varied between $0 \mathrm{~km} \mathrm{~s}^{-1}$ (thermal broadening only) and $10^{4} \mathrm{~km} \mathrm{~s}^{-1}$, an upper limit set by the observed line width. In general, the line spectrum grows stronger relative to the continuum as turbulence increases. This is because lines more easily escape due to diminished line optical depth and permitted lines are selectively strengthened by continuum pumping. Comparisons with observations reveal two cases. The predicted relative intensities of the majority of the strong lines in typical objects do not depend strongly on the microturbulent field. A turbulence of $\sim 10^{3} \mathrm{~km} \mathrm{~s}^{-1}$ does not violate observations, but is not required either. However, in the sharp-lined quasars, some lines require a turbulence of the same order as the observed line width to reproduce the spectrum.
\end{abstract}

Subject headings: galaxies: active — galaxies: nuclei — quasars: emission lines

\section{INTRODUCTION}

The nature of emission-line clouds in the broad-line region (BLR) of active galactic nuclei (AGNs) is an open question, but one that is critical for understanding their structure and physics. Early models of AGNs invoked a sea of discrete clouds moving in the vicinity of a central source of ionizing radiation. By analogy with the Crab Nebula and its resident pulsar, it was thought that AGN clouds were small discrete blobs, each intercepting a small fraction of the ionizing continuum, and then reprocessing it into line and continuum radiation (Davidson \& Netzer 1979).

Since current observations do not spatially resolve BLR clouds, information about the character of the individual BLR clouds can only come from detailed analysis of their emission lines. Spectral observations of AGNs have revealed that the lines are broad. FWHM range between $\sim 10^{3}$ and $\sim 10^{4} \mathrm{~km} \mathrm{~s}^{-1}$. The large line widths were thought to be due to the systematic movement of clouds toward or away from the continuum engine or as virial motions of clouds in a strong central gravitational potential (Rees et al. 1987). Today, however, other models such as winds from stars (Alexander \& Netzer 1994) or accretion disks (Murray \& Chiang 1998; Frank, King, \& Raine 1992), and magnetically confined filaments (Rees 1987) are being considered to describe the gas kinematics.
In addition to their great breadth, AGN emission lines are remarkably smooth. This has been interpreted as being due either to the collective, overlapping emission of very many individual clouds moving in a large velocity field or to emission from a continuous flow (Arav et al. 1997, 1998; Dietrich et al. 1999).

The primary agent thought to be responsible for a line's FWHM is the macroscopic ordered motion of the gas. Microturbulence, the motion that occurs within a cloud's line-forming region, has been assumed to be absent, with the intrinsic line width being due to thermal motions only. For hydrogen this is of the order of $V_{\mathrm{th}} \sim 10 \mathrm{~km} \mathrm{~s}^{-1}$. This is insignificant compared to the total line width. The observed line width is therefore assumed to be due to bulk motions of clouds, or macroturbulence. This leads to the conundrum that an improbably large number of clouds are needed to account for the smooth profiles (Arav et al. 1997).

Some models predict that significant microturbulence should be present. A wind can produce a large velocity gradient, which can mimic turbulence, while nondissipative MHD waves in magnetically confined clouds are another possibility (Rees 1987; Bottorff \& Ferland 2000). In the latter case, if the gravitational and magnetic fields are in overall energy equipartition, the broadening due to largescale, nondissipative microturbulence will be comparable to 
the observed line width. Electron scattering could also explain smooth broad line profiles, especially in the line wings (Emmering et al. 1992).

No matter what its origin, the presence of microturbulent line broadening within a cloud will have important effects on the emitted spectrum. This is because turbulence lowers line opacity, pushing the onset of line saturation deeper into the cloud, hence altering the line radiative transfer. Additionally, pumping by the incident continuum becomes more important as the turbulence increases and a line can absorb a larger fraction of the continuum.

What can we deduce about the turbulence within a single cloud from its observed spectrum? Shields, Ferland, \& Peterson (1995) investigated the effects of microturbulence on stronger quasar emission lines in a single cloud, and Hamann et al. (1998) discussed the effects of $1000 \mathrm{~km} \mathrm{~s}^{-1}$ turbulence and radial outflow for two ionization parameters and one density. Surprisingly, we know of no extensive studies of the effects of turbulence, even though line profile smoothness seems to require it and several of the models mentioned above should have considerable microturbulence. At first glance this seems to be only a technical issue, but strict limits to the presence or absence of microturbulence have profound implications for the nature of the clouds, the central engine, and the observed line profiles.

In order to make our results as general as possible, we do not consider any one model of the origin of the clouds or the turbulence. Microturbulence could be due to any velocity field that occurs over distances that are small compared to a photon's mean free path.

The existence of large-scale microturbulence would generate tremendous heating if it were just random turbulence. Quasar emission-line spectra, however, do not suggest profound shock heating (Ferland et al. 1996). This indicates that microturbulence, if it exists, needs to be nondissipative or occur over large scales. Nondissipative MHD waves, which seem to be ubiquitous in nature, could do this, although a significant shear or velocity gradient on the order of a photon mean free path would also mimic microturbulence.

In $\S 2$ we consider the effect of a range of microturbulences on a single AGN cloud of fixed density, exposed to a fixed ionizing continuum flux. In $\S 3$ we generalize the results to an ensemble of clouds with a wide range of density and exposed to a wide range in ionizing flux. The conclusions of $\S 2$ and $\S 3$ are similar, suggesting that the results are not strongly model dependent. In $\S 4$ we compare our results with observations and in $\S 5$ discuss our results.

\section{A SINGLE CLOUD}

\subsection{Overview}

In this section we consider the effects of microturbulence on the spectrum of a single cloud exposed to a fixed incident continuum flux. We parameterize the magnitude of microturbulence by a velocity $V_{\text {turb }}$. This is increased while density and flux are held fixed. We choose a hydrogen density $n_{\mathrm{H}}=10^{10.0} \mathrm{~cm}^{-3}$ and hydrogen ionizing photon flux $\Phi=10^{18.5} \mathrm{~cm}^{-2} \mathrm{~s}^{-1}$. These values correspond to "typical" cloud parameters (Davidson \& Netzer 1979). The cloud column density is set at $10^{23} \mathrm{~cm}^{-2}$ and the gas is assumed to have solar composition. The cloud is exposed to a power-law continuum with slope $f_{v} \sim v^{-1.2}$ and an infrared break at $0.1 \mu \mathrm{m}$. Most of these parameters are the same as those used in Korista et al. (1997a), although the spectral energy distribution is different. All of these parameters are kept fixed since we concentrate on the effects of turbulence.

\subsection{Lines We Consider}

Our photoionization models include the $\sim 10^{6}$ atomic and molecular transitions included in version 94 of the photoionization code Cloudy (Ferland 2000). From these, the intensities of $\sim 160$ emission lines were extracted and studied for possible sensitivity to $V_{\text {turb }}$. This set of lines included the lowest permitted and intercombination transitions of all of the astrophysically abundant elements $(\mathrm{H}$, $\mathrm{He}, \mathrm{C}, \mathrm{N}, \mathrm{O}, \mathrm{Ne}, \mathrm{Mg}, \mathrm{Si}, \mathrm{S}, \mathrm{Fe}$ ). Many of these lines are discussed in Korista et al. (1997a), although a few additional ones have been added. We will show results for a subset that include selected frequently measured quasar emission lines, and lines that change by appreciable amounts when microturbulence is present.

Hamann et al. (1998) discuss the effects of turbulence on BLR spectra for a few parameters. They note that the effects may be underestimated by factors of 1.5-2 due to the fact that finite model atoms are used. Excitations to high levels, followed by cascades to the levels we predict, would be missed. Similarly, the radiative transport techniques we use to include line shielding are probably uncertain by similar factors. The effects we consider below, however, are much larger.

\subsection{Microturbulence}

Microturbulence affects the spectrum through changes in a line's intrinsic width. Without microturbulence, the Doppler width $V$ is given by the thermal width $V_{\text {th }}=$ $(2 k T / m)^{1 / 2}$. Here $k$ is Boltzmann's constant, $T$ is the temperature, and $m$ is the ion's mass. Microturbulence is included in the Doppler width through

$$
V=\left(V_{\text {th }}^{2}+V_{\text {turb }}^{2}\right)^{1 / 2} \text {. }
$$

Adding a velocity field in this way is appropriate when $V_{\text {turb }}$ has a length scale that is shorter than the photon mean free path,

$$
l_{\mathrm{mfp}} \approx\left[\sigma\left(n_{A} / n_{\mathrm{H}}\right) n_{\mathrm{H}}\right]^{-1} \approx 10^{8 \pm 1}\left(\frac{10^{10}}{n_{\mathrm{H}}}\right)\left(\frac{10^{-4}}{n_{A} / n_{\mathrm{H}}}\right) \mathrm{cm} .
$$

Here $\sigma \sim 10^{-14 \pm 1} \mathrm{~cm}^{2}$ represents a typical cross-section range for a UV transition, $n_{\mathrm{H}}$ is the hydrogen particle density in $\mathrm{cm}^{-3}$, and the abundance, by number, of a typical ion relative to hydrogen is taken to be $n_{A} / n_{\mathrm{H}} \sim 10^{-4}$.

We note that if, instead of microturbulent motions, there is a continuous flow with a significant velocity gradient over the scale of $l_{\mathrm{mfp}}$ then the microturbulent Doppler broadening formula still applies. For instance, winds blown from stars will accelerate over scales comparable to the scale height of its atmosphere, and the line width caused by this shear can be considered as a form of turbulence as studied here.

The value of $l_{\operatorname{mfp}}$ is 4-6 orders of magnitude smaller than the inferred thickness, $l_{\text {BLR }}$, of "typical" broad-line region clouds:

$$
l_{\mathrm{BLR}} \approx N / n_{\mathrm{H}} \approx 10^{13}\left(\frac{N}{10^{23}}\right)\left(\frac{10^{10}}{n_{\mathrm{H}}}\right) \mathrm{cm} .
$$

Here $N$ is the cloud column density in units of $\mathrm{cm}^{-2}$. Thus the "typical" BLR cloud has room to support many individual microturbulent motions. 
A baseline calculation is first done for $V_{\text {turb }}=0 \mathrm{~km} \mathrm{~s}^{-1}$. $V_{\text {turb }}$ is then increased in 0.3 dex steps through $10^{4} \mathrm{~km} \mathrm{~s}^{-1}$. The results for various lines are shown in two tables. Table 1 gives selected lines, chosen because they are frequently measured. Table 2 gives the lines we found to be most sensitive to $V_{\text {turb }}$, having changed by more than $1.5 \mathrm{dex}$. The first column in each table gives the line label and wavelength. The second column, labeled "Single Cloud," gives the log of the ratio of equivalent width relative to the continuum at $1215 \AA$ at $V_{\text {turb }}=10^{4} \mathrm{~km} \mathrm{~s}^{-1}$ to $V_{\text {turb }}=0 \mathrm{~km} \mathrm{~s}^{-1}$. This is done to show the relative increase (or decrease) of line intensity. Four lines, Si II $\lambda 1260, \mathrm{Si}$ II $\lambda 1305, \mathrm{C}$ II $\lambda 1335$, and $\mathrm{Fe}$ II

TABLE 1

Selected Frequently Measured Quasar EMission Lines

\begin{tabular}{|c|c|c|c|c|c|c|c|c|c|c|}
\hline \multicolumn{2}{|c|}{ LINE } & \multicolumn{2}{|c|}{ SiNGle Cloud $^{\mathrm{a}}$} & \multicolumn{7}{|c|}{ STANDARD LOC MODEL } \\
\hline Ion & $\begin{array}{c}\lambda \\
(\AA)\end{array}$ & $\log$ & {$\left[\frac{\mathrm{EW}\left(10^{4}\right)}{\mathrm{EW}(0)}\right]$} & $\mathrm{EW}(0)$ & EW(10) & $\operatorname{EW}\left(10^{2}\right)$ & $\operatorname{EW}\left(10^{3}\right)$ & $\operatorname{EW}\left(10^{4}\right)$ & $\log$ & {$\left[\frac{\mathrm{EW}\left(10^{4}\right)}{\mathrm{EW}(0)}\right]$} \\
\hline $\mathrm{C}$ III ........ & 977 & & 0.86 & 15.4 & 15.0 & 19.7 & 43.9 & 165.2 & & 1.03 \\
\hline $\mathrm{N}$ III ........ & 991 & & 1.07 & 3.3 & 3.3 & 5.1 & 35.9 & 51.1 & & 1.19 \\
\hline $\operatorname{Ly} \beta \ldots \ldots$. & 1026 & & 1.03 & 5.8 & 5.7 & 5.1 & 13.9 & 70.5 & & 1.08 \\
\hline $\mathrm{O}$ vi ........ & $1032+1037$ & & 0.72 & 72.5 & 72.2 & 75.4 & 93.7 & 195.2 & & 0.43 \\
\hline $\operatorname{Ly} \alpha \ldots \ldots . .$. & 1216 & & 0.46 & 568.3 & 587.6 & 589.2 & 1342.5 & 2602.7 & & 0.66 \\
\hline Nv ........ & $1239+1243$ & & 0.45 & 14.2 & 14.1 & 15.5 & 25.1 & 60.0 & & 0.63 \\
\hline Si II........ & 1260 & & 2.64 & 0.6 & 0.6 & 3.2 & 25.6 & 158.0 & & 2.42 \\
\hline Si II........ & 1305 & & 2.88 & 0.3 & 0.3 & 2.6 & 21.5 & 98.9 & & 2.51 \\
\hline $\mathrm{C}$ II ......... & 1335 & & 1.57 & 10.5 & 10.3 & 12.5 & 38.5 & 190.8 & & 1.26 \\
\hline Si IV ........ & $1394+1403$ & & 0.50 & 16.4 & 16.2 & 18.4 & 30.9 & 88.5 & & 0.73 \\
\hline $\mathrm{O}$ Iv $] \ldots \ldots$ & 1402 & & 0.05 & 10.8 & 10.5 & 10.2 & 10.3 & 11.8 & & 0.04 \\
\hline $\mathrm{N}$ IV]...... & 1486 & & 0.08 & 8.7 & 8.5 & 8.5 & 8.7 & 9.8 & & 0.05 \\
\hline $\mathrm{C}$ iv ........ & $1548+1551$ & & 0.20 & 255.4 & 261.1 & 287.5 & 326.7 & 506.0 & & 0.30 \\
\hline He II ....... & 1640 & & 0.37 & 40.0 & 38.3 & 37.2 & 40.6 & 78.9 & & 0.30 \\
\hline $\mathrm{O}$ III] $]. . .$. & $1661+1666$ & & 0.00 & 25.6 & 25.0 & 24.1 & 23.9 & 32.7 & & 0.11 \\
\hline $\mathrm{N}$ III] $]. . .$. & 1750 & & -0.08 & 4.3 & 4.1 & 3.8 & 3.6 & 3.7 & & -0.07 \\
\hline $\mathrm{Fe}$ II ........ & UV191 1787 & & 2.60 & 2.6 & 2.5 & 5.2 & 27.3 & 162.6 & & 1.79 \\
\hline Si II........ & 1808 & & 1.16 & 7.2 & 6.6 & 8.5 & 17.5 & 35.5 & & 0.69 \\
\hline $\mathrm{Al} \mathrm{III} \ldots . .$. & $1855+1863$ & & 1.03 & 3.7 & 3.6 & 5.1 & 14.0 & 18.7 & & 0.70 \\
\hline $\mathrm{Si}$ III] $]. . .$. & 1892 & & -0.04 & 24.2 & 23.5 & 20.6 & 20.0 & 21.5 & & -0.05 \\
\hline $\mathrm{C}$ iII $] \ldots \ldots$ & 1909 & & -0.06 & 28.7 & 28.2 & 25.8 & 25.0 & 27.1 & & -0.02 \\
\hline $\mathrm{N}$ II] $\ldots . . .$. & 2140 & & -0.08 & 0.3 & 0.2 & 0.3 & 0.2 & 0.2 & & -0.17 \\
\hline Mg II ....... & $2796+2804$ & & 0.51 & 217.2 & 302.1 & 342.0 & 342.2 & 578.6 & & 0.43 \\
\hline $\mathrm{H} \beta \ldots \ldots \ldots$ & 4861 & & 0.39 & 57.7 & 56.5 & 54.1 & 42.7 & 77.5 & & 0.13 \\
\hline
\end{tabular}

${ }^{\text {a } V a l u e s ~ f o r ~}\left[\log \left(n_{\mathrm{H}}\right), \log (\Phi)\right]$ fixed at $(10.0,18.5)$.

TABLE 2

LINES WITH $\log \left[\mathrm{EW}\left(10^{4}\right) / \mathrm{EW}(0)\right] \geq 1.5^{\mathrm{a}}$

\begin{tabular}{|c|c|c|c|c|c|c|c|c|c|c|}
\hline \multicolumn{2}{|c|}{ LiNE } & \multicolumn{2}{|c|}{ Single Cloud $^{\mathrm{b}}$} & \multicolumn{7}{|c|}{ STANDARD LOC MODEL } \\
\hline Ion & $\begin{array}{c}\lambda \\
(\AA)\end{array}$ & $\log$ & {$\left[\frac{\mathrm{EW}\left(10^{4}\right)}{\mathrm{EW}(0)}\right]$} & $\mathrm{EW}(0)$ & $\mathrm{EW}(10)$ & $\operatorname{EW}\left(10^{2}\right)$ & $\operatorname{EW}\left(10^{3}\right)$ & $\mathrm{EW}\left(10^{4}\right)$ & $\log$ & {$\left[\frac{\mathrm{EW}\left(10^{4}\right)}{\mathrm{EW}(0)}\right]$} \\
\hline $\mathrm{O} v \ldots \ldots$ & 630 & & 2.13 & 2.0 & 2.0 & 3.2 & 14.9 & 82.9 & & 1.62 \\
\hline S IV ....... & 655 & & 2.33 & 0.1 & 0.1 & 0.9 & 5.2 & 21.1 & & 2.32 \\
\hline $\mathrm{N}$ III ...... & 678 & & 1.95 & 0.1 & 0.1 & 1.1 & 5.7 & 18.7 & & 2.27 \\
\hline O III ...... & 698 & & 2.44 & 0.2 & 0.3 & 1.6 & 9.4 & 52.5 & & 2.41 \\
\hline S IV ....... & 737 & & 2.12 & 0.5 & 0.1 & 0.8 & 3.7 & 13.2 & & 1.42 \\
\hline $\mathrm{N}$ iII $\ldots \ldots$. & 752 & & 1.65 & 0.1 & 0.2 & 0.7 & 2.4 & 4.9 & & 1.60 \\
\hline $\mathrm{N}$ iv $\ldots \ldots$ & 765 & & 1.99 & 0.2 & 0.5 & 1.7 & 8.5 & 45.2 & & 2.35 \\
\hline$S \mathrm{v} \ldots \ldots \ldots$ & 786 & & 1.95 & 0.7 & 0.2 & 1.0 & 5.0 & 25.0 & & 1.55 \\
\hline H I ........ & 973 & & 1.67 & 1.0 & 1.1 & 1.7 & 7.8 & 42.0 & & 1.62 \\
\hline $\mathrm{N}$ II ....... & 1085 & & 2.52 & 0.6 & 0.6 & 2.6 & 18.3 & 67.0 & & 2.05 \\
\hline Fe III...... & 1122 & & 1.91 & 0.2 & 0.2 & 0.9 & 2.9 & 5.2 & & 1.41 \\
\hline Si II ....... & 1260 & & 2.64 & 0.6 & 0.6 & 3.2 & 25.6 & 158.0 & & 2.42 \\
\hline Si II ........ & 1305 & & 2.88 & 0.3 & 0.3 & 2.6 & 21.5 & 98.9 & & 2.51 \\
\hline $\mathrm{C}$ II ........ & 1335 & & 1.57 & 10.5 & 10.3 & 12.5 & 38.5 & 190.8 & & 1.26 \\
\hline Si II ........ & 1527 & & 2.56 & 0.8 & 0.8 & 3.0 & 22.5 & 113.9 & & 2.15 \\
\hline $\mathrm{Fe}$ II ....... & UV191 1787 & & 2.60 & 2.6 & 2.5 & 5.2 & 27.3 & 162.6 & & 1.79 \\
\hline $\mathrm{Al}$ II ........ & 1671 & & 2.38 & 1.3 & 1.1 & 3.0 & 21.5 & 113.3 & & 1.94 \\
\hline
\end{tabular}

${ }^{\text {a }}$ Values for C II 1335, Fe II 1787, Si II 1260, and Si II 1305 also appear in Table 1.

${ }^{\mathrm{b}}$ Values for $\left[\log \left(n_{\mathrm{H}}\right), \log (\Phi)\right]$ fixed at $(10.0,18.5)$. 
$\lambda 1787$, appear in both tables. The fact that they are both measurable and very sensitive to turbulence means that they will be particularly good diagnostics of turbulence.

We do not explore the effects of turbulence on the full $\mathrm{Fe}$ II spectrum. The $\mathrm{Fe}$ II $\lambda 1787$ feature is singled out because it is a strong, easily measured, feature in some objects. Verner et al. (1999) discuss some effects of turbulence on the full $\mathrm{Fe}$ in spectrum.

\subsection{Consequences of Turbulence}

Increasing $V_{\text {turb }}$ has two major effects on the spectrum. First, the line optical depths decrease as a line's opacity spreads over a larger frequency range. The result is that lines escape more freely and they become brighter. The second effect is that direct optical pumping by the incident continuum, as treated in Ferland (1992), becomes more important. This is because a line only efficiently absorbs the continuum over its Doppler width, so that as $V_{\text {turb }}$ increases, continuum pumping does too. These effects occur for all permitted lines, but the far-ultraviolet (FUV) lines, primarily excited by pumping with the incident continuum, are selectively more enhanced. Collisional excitation (a significant excitation mechanism for UV lines) is inefficient for FUV lines because they have a much higher excitation potential than the mean kinetic energy of the free electrons in the gas.

All lines change by some extent, if only because the overall temperature of a cloud changes with increasing microturbulence. The temperature tends to be lower at very large $V_{\text {turb }}$ because line photons escape more readily and cool the cloud more efficiently. Intermediate values of $V_{\text {turb }}$ produce a hotter cloud, due to continuum pumping of lines followed by their thermalization.
Intercombination lines such as C III] $\lambda 1909$ change because of the change in temperature. Continuum pumping is unimportant for intercombination lines because they tend to have small optical depths. The ratio of FUV to intercombination lines of the same ion (for instance, C III 2977 / $\mathrm{C}$ III] 21909 ) therefore tends to be a strong function of $V_{\text {turb }}$ while having no direct dependence on the ionization or composition of the cloud. These ratios are ideal candidates for indicators of microturbulence, provided that the cloud density is below the critical density of the intercombination line (typically $10^{10} \mathrm{~cm}^{-3}$ ).

Tables 1 and 2 show that most lines become brighter at large $V_{\text {turb }}$. The effects of microturbulence on the equivalent widths of Table 1 are shown graphically in Figure 1. In the figure microturbulence is increased from 0 to $10^{4} \mathrm{~km} \mathrm{~s}^{-1}$ and the lines are presented relative to their equivalent width at $0 \mathrm{~km} \mathrm{~s}^{-1}$. Because most of the lines tend to increase by the same amount ( $\sim 1$ dex), their relative ratios will not be particularly good indicators of turbulence. Some exceptions are $\mathrm{Si}$ II $\lambda 1260, \mathrm{Si}$ II $\lambda 1305, \mathrm{C}$ II $\lambda 1335, \mathrm{Si}$ II $\lambda 1260$, and $\mathrm{Fe}$ II $\lambda 1787$, for which the increase is much larger. Although the changes are great, the lines are very weak, and so not subject to the thermostat effect. Figure 2 shows the intensities of these lines relative to intercombination lines and the Si II $\lambda 1808$ line, which has a similarly weak response to microturbulence. The ratios increase by 1.0 to $2.0 \mathrm{dex}$ as $V_{\text {turb }}$ increases from 0 to $10^{4} \mathrm{~km} \mathrm{~s}^{-1}$ making them reasonable turbulence-sensitive indicators. Note that five of the six line ratios involve the same ion and are therefore abundance independent. In $\S 5$ we hope to use these to measure turbulence in some objects.

Ratios involving permitted lines have the advantage of little direct dependence on density. It turns out that Si II is

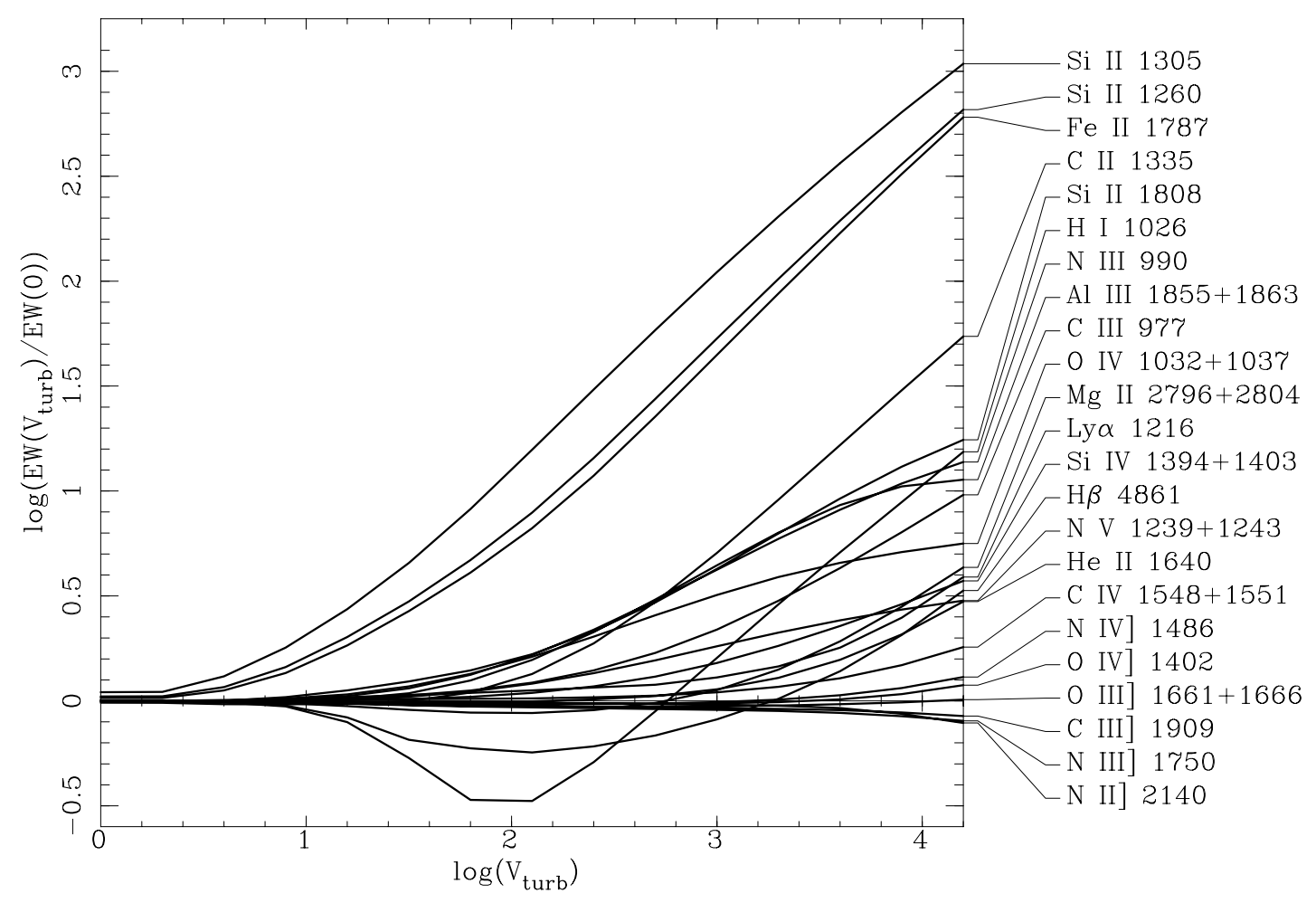

FIG. 1.-The dependence on $\log \left(V_{\text {turb }}\right)$ of frequently measured quasar emission lines of the single-cloud model. Intensities are normalized to the value when turbulence is not present. Most lines grow stronger with increasing $\log \left(V_{\text {turb }}\right)$. 


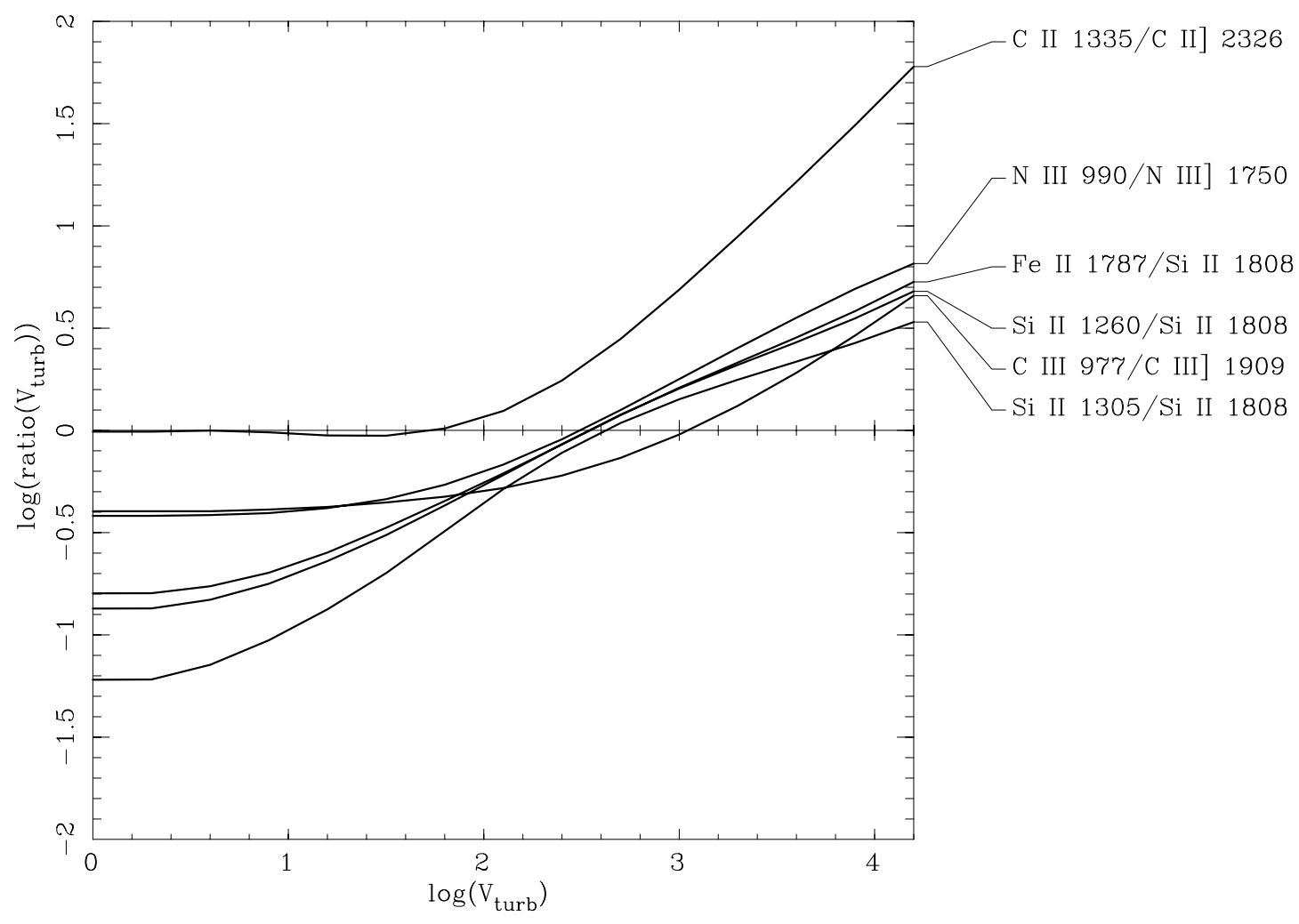

FIG. 2.-Microturbulence sensitive line ratios as a function of $\log \left(V_{\text {turb }}\right)$. The actual value of the ratio is shown.

the only ion where this comparison is possible (§ 2.2), so we had to use intercombination lines in the other cases. Unfortunately the intercombination to resonance line ratios are also a function of density, because the lines have very different critical densities, and temperature, due to their different excitation potentials. Higher than expected densities could mimic turbulence because the intercombination lines will be weakened relative to the permitted lines. In the next section we consider a distribution of clouds with a broad mix of densities as a check on density effects.

\section{LOCALLY OPTIMALLY EMITTING CLOUD MODELS}

The analysis presented above is only for a single cloud, of fixed density, exposed to a single ionizing flux. While this single-cloud model is illustrative, real AGNs are likely to have clouds with a wide variety of density. In addition, reverberation analysis shows that AGN are ionization stratified, so clouds must also be subject to a range in ionizing continuum flux (Peterson 1993). It is therefore important to examine the effects of microturbulence on global (in $n_{\mathrm{H}}$ and $\Phi$ phase space) models of the AGN environment to determine whether conclusions for a single cloud continue to be valid. To this end we consider the locally optimally emitting clouds (LOC) model of Baldwin et al. (1995). In $\S 4$ we compare it with the single-cloud calculations of $\S 2$ and with observation.

We examine this class of models since it is a simple example of a distributed geometry and shows how a mixture of clouds can affect the ratios we discuss. At each radius there is assumed to be a population of clouds with a broad mix of density and therefore an equally wide range in ionization parameter. A combination of atomic physics and radiative transport will assure that some of the clouds have optimal conditions for the emission of a particular line.
These clouds will then dominate the contribution to the emission-line flux. The line luminosities are determined by integrating over a distribution of cloud properties, giving

$$
L_{\text {line }} \propto \iint r^{2} F(r) f(r) g\left(n_{\mathrm{H}}\right) d n_{\mathrm{H}} d r .
$$

Here $F(r)$ is the predicted line flux of a cloud at radius $r$, $f(r)$ is the radial distribution of the covering factor, and $g\left(n_{\mathrm{H}}\right)$ gives the density distribution $n_{\mathrm{H}}$ at each radius. The density limits for the integration are $10^{8}$ and $10^{14} \mathrm{~cm}^{-3}$, and the limits for the ionizing photon flux $\Phi$ are $10^{18}$ and $10^{24} \mathrm{~cm}^{-2} \mathrm{~s}^{-1}$, as described in Baldwin et al. (1995). We choose $f(r) \propto$ constant and $g\left(n_{\mathrm{H}}\right) \propto n_{\mathrm{H}}^{-1}$ in accord with the original parameterization of Baldwin et al. (1995). We note that further modeling, using $f(r) \propto r^{\gamma}$, indicates a mild dependence of the line strengths on $\gamma$ where $-1<\gamma<1$ (Baldwin 1997; Korista et al. 1998; Korista \& Goad 2000). Here however, we focus on the effects of large-scale turbulence and do not vary these other parameters.

The effects of microturbulence were investigated by increasing internal cloud turbulence in 1 dex increments from 1 to $10^{4} \mathrm{~km} \mathrm{~s}^{-1}$. The microturbulence was assumed to be uniform across the $\log \left(n_{\mathrm{H}}\right), \log (\Phi)$ plane. This produced a three-dimensional "data cube" for each emission line in the $\log \left(n_{\mathrm{H}}\right), \log (\Phi), V_{\text {turb }}$ phase space. An LOC model from a grid with no turbulence was also calculated for comparison.

\subsection{LOC Model Results}

Table 1 gives equivalent widths for selected bright quasar lines. These were predicted assuming full coverage by clouds. We refer to these models as "standard LOC models" to emphasize the fact that they are the Baldwin et al. (1995) model with the microturbulent velocity held constant across the LOC plane. The last column of the table 


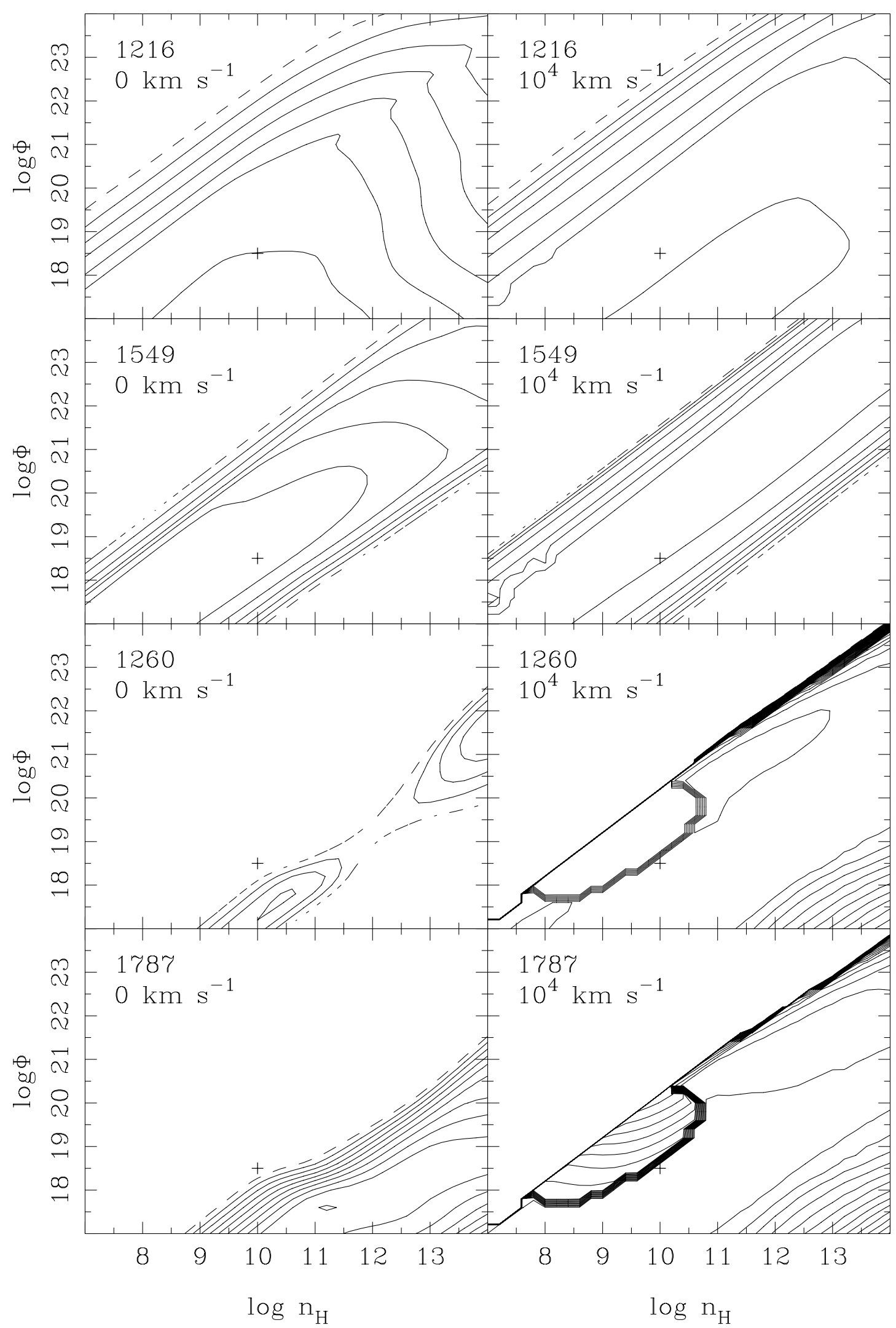

FIG. 3. - Contours of $\log (\mathrm{EW})$ for four emission lines ( $\mathrm{Ly} \alpha \lambda 1216, \mathrm{C}$ IV $\lambda 1549, \mathrm{Si}$ II $\lambda 1260$, and Fe II $\lambda 1787$ ), referenced to the incident continuum at $1215 \AA$, are shown as functions of the hydrogen density and ionizing photon flux. Each value of $\mathrm{EW}$ within the $\log \left(n_{\mathrm{H}}\right), \log (\Phi)$ plane assumes full source coverage. The thermal broadening case, $V_{\text {turb }}=0 \mathrm{~km} \mathrm{~s}^{-1}$, is on the left. The $V_{\text {turb }}=10^{4} \mathrm{~km} \mathrm{~s}^{-1}$ case is on the right. For a given line, contours have equal levels of log(EW). The lowest contour level (dashed contour) corresponds to a $1 \AA \mathrm{EW}$ relative to the continuum at $1215 \AA$. Subsequent contour levels are increased by 0.5 dex for $\mathrm{Ly} \alpha \lambda 1216$, and C IV $\lambda 1549$ and $0.1 \mathrm{dex}$ for $\mathrm{Si}$ II $\lambda 1260$, and Fe II $\lambda 1787$. The extra contours in the $V_{\text {turb }}=10^{4} \mathrm{~km} \mathrm{~s}^{-1}$ plots, as compared to the $V_{\mathrm{turb}}=0 \mathrm{~km}$ $\mathrm{s}^{-1}$ plots, reveal the increase in EW with $V_{\text {turb }}$. Crosses show the location of our "Single Cloud" calculation in the $\log \left(n_{\mathrm{H}}\right), \log (\Phi)$ plane. 
gives the log of the increase in $\mathrm{EW}$ between the $V_{\text {turb }}=$ $0-10^{4} \mathrm{~km} \mathrm{~s}^{-1}$ standard LOC models. There is a clear increase in the equivalent width of most of the lines. The trend is very similar to the results for the single-cloud calculation, suggesting that the single cloud is a good predictor of the behavior of a global distribution of clouds. The fact that the results are similar is not too surprising: the strong selection effects identified by Baldwin et al. (1995) also come into play when microturbulence is present as well.

Table 2 is similar to Table 1 but lists the equivalent widths of lines that increase by more than 1.5 dex [for the single $\log \left(n_{\mathrm{H}}\right)=10.0, \log (\Phi)=18.5$ cloud $]$. The change in the equivalent widths of the standard LOC models between $V_{\text {turb }}=0 \mathrm{~km} \mathrm{~s}^{-1}$ and $V_{\text {turb }}=10^{4} \mathrm{~km} \mathrm{~s}^{-1}$ for these lines, as with the bright quasar lines, is comparable with the changes in the single cloud calculation. Two noteworthy changes that occur in this table are $\mathrm{Al}$ II $\lambda 1671$ because it may influence the He II $\lambda 1640+\mathrm{O}$ III] $\lambda 1663+\mathrm{Al}$ II $\lambda 1671$ blend, and the occasionally observed Si II $\lambda 1527$ line. Nondetection of these lines may therefore provide an upper limit to turbulence.

The predicted equivalent widths of a few lines are shown in Figure 3. The figure shows Ly $\alpha, \mathrm{C}$ IV $\lambda 1549$, Si II $\lambda 1260$, and Fe II $\lambda 1787$, for $V_{\text {turb }}$ of 0 and $10^{4} \mathrm{~km} \mathrm{~s}^{-1}$. Microturbulence tends to produce a global increase in emission and makes the lines relatively stronger at large values of $\log \left(n_{\mathrm{H}}\right)$ and $\log (\Phi)$ (the upper right corner of the contour plots of Fig. 3). This is due to the diminished effects of line thermalization. Note that while Ly $\alpha$ and C IV $\lambda 1549$ increase, the lines Si II $\lambda 1260$ and Fe II $\lambda 1787$ increase even more.

\subsection{An Equipartition Model}

In addition to the standard LOC model described above, we investigated a model in which the kinetic energy of microturbulent motion and the gravitational potential are in energy equipartition. This is a natural consequence of equipartition between magnetic and gravitational fields (Bottorff \& Ferland 2000), as would happen for magnetically confined clouds (Rees 1987). The density and flux weighting functions used in the previous LOC models were used here too. This model will be referred to as the LOC equipartition model.

Energy equipartition implies that $V_{\text {turb }}^{2} \propto R^{-1}$ which in turn implies that $V_{\text {turb }} \propto \Phi^{1 / 4}$. The model microturbulence is normalized so that $V_{\text {turb }}=10^{4} \mathrm{~km} \mathrm{~s}^{-1}$ when $\Phi=10^{24}$ $\mathrm{cm}^{-2} \mathrm{~s}^{-1}$. This normalization produces $V_{\mathrm{turb}} \sim 300 \mathrm{~km} \mathrm{~s}^{-1}$ at $\Phi=10^{18} \mathrm{~cm}^{-2} \mathrm{~s}^{-1}$ which is consistent with the transition, in velocity space, between the broad emission line clouds we are discussing here and the occasionally resolved narrow emission line clouds. In $\S 4$ we compare this model and the standard LOC models with each other and with observation.

\section{COMPARISON OF MICROTURBULENT CLOUDS WITH OBSERVATIONS}

Our goal is either to calibrate model-independent line ratios that directly measure cloud turbulence or, at least, to understand what limits the spectrum can place on the velocity field. Previous work has shown that many lines can be reproduced without turbulence, but some line ratios are not reproduced. In particular, in one of the few QSOs with good enough data to obtain high-quality measurements of the lines in Table 2, the observed ratio of $\mathrm{Si}$ II $\lambda 1260$ to $\mathrm{Si}$ II $\lambda 1808$ is several orders of magnitude larger than is predicted (Baldwin et al. 1996). Simulations presented in that paper suggested that the small ratio was caused by underprediction of Si II $\lambda 1260$ rather than overprediction of Si II $\lambda 1808$.

Note that all but a few of the bright quasar lines in Table 1 increase in equivalent width with increasing $V_{\text {turb }}$, with the most dramatic increases occurring in Si II $\lambda 1260$, Si II $\lambda 1305$, and $\mathrm{Fe}$ II $\lambda 1787$. These lines increase by $2.42,2.51$, and 1.79 dex, respectively (col. [9] of Table 1). The large increase in Si II $\lambda 1260$ suggests that microturbulence may help account for observations of this line in some objects.

The overall increase in intensity of the emission lines is also important. Korista, Ferland, \& Baldwin (1997b) showed that the observed quasar FUV continuum is too soft to account for the observed intensities of prominent lines. They suggested that the continuum viewed by the clouds may be harder than the continuum we observe. In the following we shall refer to this as the He II energy budget problem. The results presented here may offer a partial alternative explanation - the overall line spectrum grows stronger with increasing $V_{\text {turb }}$ so the observed continuum might be consistent with the line equivalent widths if significant microturbulence is present.

In order to quantify these comparisons, $\S 4.1$ and $\S 4.2$ compare predicted line ratios to values measured from a composite spectrum (Zheng et al. 1997) and to the range of ratios measured in a few particularly well observed objects (Baldwin et al. 1996). The composite spectrum has high signal to noise, but has the drawback that it includes many QSOs with broad emission lines, so that the weak lines of interest are washed out by the continuum and often blended with other emission or absorption features. The individual objects in Baldwin et al. (1996) were selected for their narrower emission lines, which improve the detection limit for weak lines but means that these may be atypical objects.

\subsection{Line Ratios Relative to Ly}

Table 3 shows the strength of various emission lines and emission-line blends relative to $\mathrm{Ly} \alpha$ for standard LOC models with $V_{\text {turb }}=0,10,10^{2}, 10^{3}$, and $10^{4} \mathrm{~km} \mathrm{~s}^{-1}$ as well as for LOC equipartition model. The models show relatively good agreement (factor of $\sim 2$ ) with most of the observed lines except for the $\mathrm{N} v \lambda 1239+\mathrm{N} v \lambda 1243$ blend, which is under predicted. We have argued elsewhere that differences between the observed $\mathrm{N} v \lambda 1239+\mathrm{N}$ v $\lambda 1243$ blend and the models are due to enhanced quasar metallicity (see Korista, Baldwin, \& Ferland 1998 and the review by Hamann \& Ferland 1999).

Between $V_{\text {turb }}=10$ and $V_{\text {turb }}=10^{3} \mathrm{~km} \mathrm{~s}^{-1}$ all the lines or line blends relative to $\mathrm{Ly} \alpha$ change in a monotonic manner. In this range the table shows that, longward of $1000 \AA$, lines tend to decrease relative to $\mathrm{Ly} \alpha$ with increasing turbulence. With the exception of the $\mathrm{Mg}$ II to $\mathrm{Ly} \alpha$ ratio, which crosses its observed range between $10^{2}$ and $10^{3} \mathrm{~km} \mathrm{~s}^{-1}$, the calculated ratios fall away from the observed ranges with increasing turbulence. For example, the ratio $\mathrm{H} \beta / \mathrm{Ly} \alpha$, begins barely within the observed range, but drops below the observed range for values of $V_{\text {turb }}$ above $10^{2} \mathrm{~km} \mathrm{~s}^{-1}$. This suggests that little microturbulence is present. On the other hand, line blends shortward of $1000 \AA$ increase relative to Ly $\alpha$ moving predictions toward observed values, and arguing for microturbulence. We conclude from this that the presence of high degrees of microturbulence cannot be 
TABLE 3

Line Strength Relative to Ly $\alpha$ : Observation and Models

\begin{tabular}{|c|c|c|c|c|c|c|c|c|}
\hline \multirow[b]{2}{*}{ EMISSION LiNe BLEND } & \multicolumn{2}{|c|}{ OBSERVED } & \multicolumn{5}{|c|}{ STANDARD LOC MODEL } & \multirow{2}{*}{$\begin{array}{c}\text { EQUIPARTITION } \\
\text { MODEL }\end{array}$} \\
\hline & Zheng & Baldwin & $0^{\mathrm{a}}$ & 10 & $10^{2}$ & $10^{3}$ & $10^{4}$ & \\
\hline $\mathrm{O}$ III $\lambda 835+\mathrm{O}$ II $\lambda 834 \ldots$. & 0.014 & & 0.005 & 0.005 & 0.006 & 0.009 & 0.016 & 0.009 \\
\hline $\mathrm{C}$ III $\lambda 977+\operatorname{Ly} \gamma \lambda 973 \ldots \ldots \ldots \ldots .$. & 0.009 & $0.007-0.20$ & 0.03 & 0.03 & 0.02 & 0.04 & 0.08 & 0.04 \\
\hline $\mathrm{N}$ III $\lambda 990$.................................... & 0.011 & 0.013 & 0.01 & 0.01 & 0.01 & 0.01 & 0.02 & 0.01 \\
\hline $\mathrm{O}$ vi $\lambda 1032+\mathrm{O}$ vi $\lambda 1037+\operatorname{Ly} \beta \lambda 1026 \ldots \ldots \ldots$ & 0.190 & $0.068-0.69$ & 0.14 & 0.13 & 0.09 & 0.08 & 0.10 & 0.09 \\
\hline $\mathrm{N}$ v $\lambda 1239+\mathrm{N}$ v $\lambda 1243 \ldots \ldots \ldots \ldots \ldots \ldots \ldots \ldots \ldots \ldots \ldots \ldots \ldots \ldots$ & 0.110 & $0.069-0.99$ & 0.03 & 0.04 & 0.02 & 0.02 & 0.02 & 0.02 \\
\hline Si IV $\lambda 1394+$ Si IV $\lambda 1403+$ O IV $] \lambda 1402 \ldots \ldots$ & 0.075 & $0.022-0.50$ & 0.05 & 0.05 & 0.03 & 0.03 & 0.04 & 0.03 \\
\hline C IV $\lambda 1548+C$ iv $\lambda 1551 \ldots \ldots \ldots \ldots \ldots \ldots \ldots$ & 0.620 & $0.087-0.65$ & 0.45 & 0.44 & 0.33 & 0.24 & 0.19 & 0.28 \\
\hline He II $\left.\lambda 1640+\mathrm{O}_{\mathrm{III}}\right] \lambda 1663+\mathrm{Al}$ II $\lambda 1671 \ldots \ldots$ & 0.068 & $0.013-0.14$ & 0.12 & 0.11 & 0.08 & 0.06 & 0.08 & 0.06 \\
\hline $\mathrm{C}$ III] $\lambda 1909+\mathrm{Si}$ III $] \lambda 1892+\mathrm{Al}$ III $\lambda 1859 \ldots \ldots$ & 0.163 & $0.076-0.74$ & 0.10 & 0.09 & 0.06 & 0.04 & 0.03 & 0.05 \\
\hline Mg II $\lambda 2796+\operatorname{Mg}$ II $\lambda 2804 \ldots \ldots \ldots \ldots \ldots \ldots \ldots$ & 0.250 & $0.15-0.30^{\mathrm{b}}$ & 0.38 & 0.51 & 0.40 & 0.25 & 0.22 & 0.30 \\
\hline 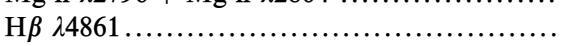 & $\ldots$ & $0.07-0.20^{\mathrm{b}}$ & 0.10 & 0.10 & 0.06 & 0.03 & 0.04 & 0.04 \\
\hline 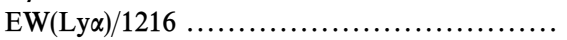 & 0.076 & $0.03-0.20$ & 0.47 & 0.48 & 0.71 & 1.10 & 2.14 & 0.95 \\
\hline
\end{tabular}

${ }^{\mathrm{a}}$ Microturbulence in units of $\mathrm{km} \mathrm{s}^{-1}$.

${ }^{\mathrm{b}}$ Ranges for observations are from Baldwin et al. 1995.

ruled out on the basis of the line ratios, relative to $\mathrm{Ly} \alpha$, presented in Table 3 alone.

Above $V_{\text {turb }}=10^{3} \mathrm{~km} \mathrm{~s}^{-1}$ five of the line blends increase again. An example is the $\mathrm{He}$ II $\lambda 1640+\mathrm{O}$ III] $\lambda 1663+\mathrm{Al} \mathrm{II}$ $\lambda 1671$ blend. In the standard LOC model the blend decreases from 0.12 at $V_{\text {turb }}=0 \mathrm{~km} \mathrm{~s}^{-1}$ to 0.06 at $V_{\text {turb }}=$ $10^{3} \mathrm{~km} \mathrm{~s}^{-1}$ but then increases to 0.08 at $V_{\text {turb }}=10^{4} \mathrm{~km} \mathrm{~s}^{-1}$. The reason for the increase is because, in the model, the contribution of $\mathrm{Al}$ II $\lambda 1671$ grows rapidly between $10^{3}$ and $10^{4} \mathrm{~km} \mathrm{~s}^{-1}$ (see Table 2) and more than compensates for the increase in $\mathrm{Ly} \alpha$ in the denominator of the line blend ratio.

Observationally the $\mathrm{He}$ II $\lambda 1640$ line and the $\mathrm{O}$ III] $\lambda 1663$ line are detected but the $\mathrm{Al}$ II $\lambda 1671$ line is not. This places a constraint of $V_{\text {turb }} \leq 10^{3} \mathrm{~km} \mathrm{~s}^{-1}$ on microturbulence in the standard LOC model. The reason is that for $V_{\text {turb }}>10^{3} \mathrm{~km}$ $\mathrm{s}^{-1}$ the predicted line intensity of $\mathrm{Al}$ II $\lambda 1671$ is comparable to the intensity of $\mathrm{He}$ II $\lambda 1640$ and $\mathrm{O}$ III] $\lambda 1663$ and therefore should be observed. A similar increase occurs for Al III $\lambda 1859$ (this line is observed) in the C III] $\lambda 1909+\mathrm{Si} \mathrm{III]}$ $\lambda 1892+\mathrm{Al}$ III $\lambda 1859$ blend but is insufficient to halt the decrease in the blend ratio by $V_{\text {turb }}=10^{4} \mathrm{~km} \mathrm{~s}^{-1}$. Nevertheless Al III $\lambda 1859$ quadruples in strength as $V_{\text {turb }}$ is increased from 0 to $10^{3} \mathrm{~km} \mathrm{~s}^{-1}$ becoming comparable to C III] $\lambda 1909$ and Si III] $\lambda 1892$ for $V_{\text {turb }}=10^{2}-V_{\text {turb }}=10^{3} \mathrm{~km} \mathrm{~s}^{-1}$.

All of our models were calculated for a cumulative covering factor of 1 . The normalized Ly $\alpha \mathrm{EW}$ is shown at the bottom of Table 3. Over the full range of microturbulence the Ly $\alpha \mathrm{EW}$ increases by a factor of about 4.6. Comparison of all the LOC models with the observed Ly $\alpha$ EW suggests the models require a modest global covering fraction in the range of $\sim 0.01$ to $\sim 0.43$. The presence of very large microturbulent velocities $\left(V_{\text {turb }}>1000 \mathrm{~km} \mathrm{~s}^{-1}\right)$ could help solve the He II energy budget problem (Korista et al. 1997b). This may suggest a mix of turbulent velocities, such as exists in the equipartition LOC model (see discussion in $\S 4.2$ below).

We note that the data in Brotherton et al. (1994) show a correlation between width and intensity ratio within components of the $\mathrm{C} \mathrm{IV} / \mathrm{Ly} \alpha$ line. The sense of the correlation in that paper is actually the opposite of what we would predict for the constant turbulence models if the observed line width indicates turbulence. The correlation in Brotherton et al. (1994) is, however, understandable in terms of the equipartition model because high-velocity regions are more highly ionized so there is a correlation between ionization and turbulence.

\subsection{Turbulence Sensitive Line Ratios and Quasars}

The ideal microturbulence indicator would be a pair of lines that are insensitive to composition, density, and ionization, but are affected by $V_{\text {turb }}$. The line ratios in Table 3 are not particularly sensitive to $V_{\text {turb }}$. A few line ratios were found to be strong functions of $V_{\text {turb }}$ for the standard LOC model. These ratios were identified by the single cloud calculations of $\S 2$ and are C II $\lambda 1335 / \mathrm{C}$ II] $\lambda 2326, \mathrm{~N}$ III $\lambda 990 /$ $\mathrm{N}$ III] $\lambda 1750, \mathrm{C}$ III $\lambda 977 / \mathrm{C}$ III] $\lambda 1909, \mathrm{Fe}$ II $\lambda 1787 / \mathrm{Si}$ II $\lambda 1808$, Si II $\lambda 1260 / \mathrm{Si}$ II $\lambda 1808$, and $\mathrm{Si}$ II $\lambda 1305 / \mathrm{Si}$ II $\lambda 1808$. Figure 4 shows a graph of these ratios as a function of $\log \left(V_{\text {turb }}\right)$. The Figure is similar to Figure 2 but for the standard LOC model rather than a single cloud.

As discussed in $\S 3$, with the exception of $\mathrm{Fe}$ II $\lambda 1787 / \mathrm{Si}$ II $\lambda 1808$, all ratios are for lines of the same ion and are therefore abundance and ionization independent. The abundance dependent $\mathrm{Fe}$ II $\lambda 1787 / \mathrm{Si}$ II $\lambda 1808$ ratio was added since we previously found that photoionization models, with thermal broadening only, have difficulty reproducing it (Baldwin et al. 1996). All of these ratios increase by factors between $\sim 10$ and $10^{2}$ and might be powerful signatures of microturbulence.

The line ratios of Figure 4, when compared with observed ratios, can be used to estimate the microturbulent field. The figure illustrates an important point about the equipartition model. The LOC equipartition model possesses microturbulent velocity elements ranging up to $10^{4} \mathrm{~km} \mathrm{~s}^{-1}$ but produces calculated line ratios that, if interpreted as a standard LOC model, yield a value of $V_{\text {turb }}$ between $10^{2}$ and $10^{3}$ $\mathrm{km} \mathrm{s}^{-1}$ (also compare the equipartition model with the standard LOC models of Table 3). This is shown in Figure 4 where the star-shaped graph markers show the positions of the line ratios of the equipartition model imposed on the standard LOC model. The reason for under prediction of the level of microturbulence is that the weighting scheme $f(r)$ places significant weight on emission from the outer boundary, where the value of $V_{\text {turb }}$ is small $\left(300 \mathrm{~km} \mathrm{~s}^{-1}\right)$. This graphical exercise shows that the contribution to emission by low $V_{\text {turb }}$ gas can dominate the total line strength even though very high $V_{\text {turb }}$ gas is present. Line ratios there- 


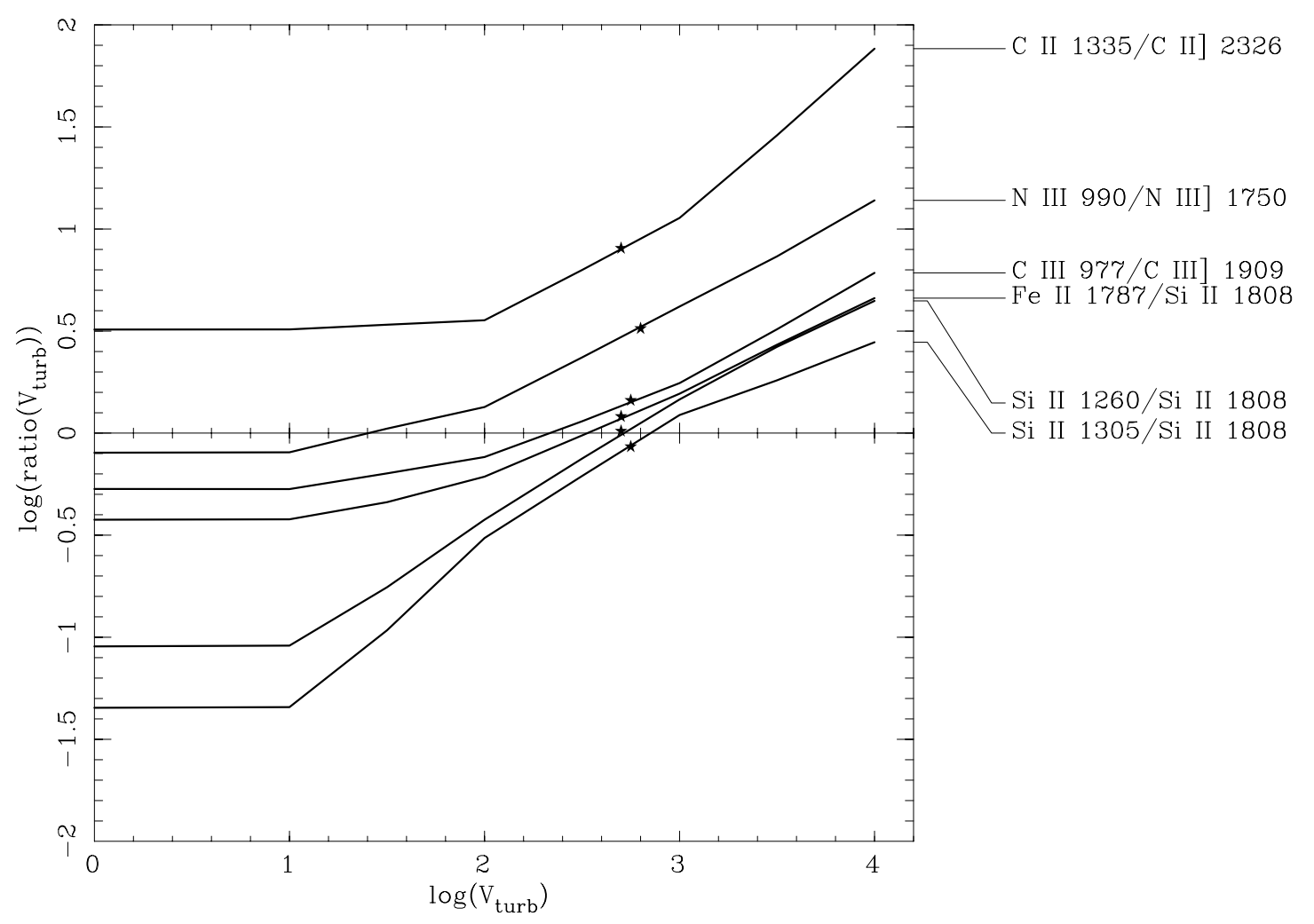

FIG. 4. - Same as Fig. 2 but for the LOC integration. The equipartition case, mapped onto the standard LOC model, is shown as stars.

fore indicate lower levels of microturbulence rather than actually present and extremely high levels of microturbulence may exist. This effect could account for the nondetection of Al II $\lambda 1671$ and the weak detection of Al III $\lambda 1859$ in observations.

Unfortunately $\mathrm{He}$ II $\lambda 1640$ is also reduced in the equilibrium model. The result is that the He II $\lambda 1640$ line in the equipartition model is smaller than in the standard LOC model with highest $\left(\sim 10^{4} \mathrm{~km} \mathrm{~s}^{-1}\right)$ microturbulence. Thus the He II energy budget problem is not solved by high levels of microturbulence because Al II $\lambda 1671$ and Al III $\lambda 1859$ cannot be eliminated or reduced while simultaneously boosting the He II $\lambda 1640$ to Ly $\alpha$ line ratio. To resolve this problem an elevation of $\mathrm{He}^{++}$producing radiation is probably still needed to explain the strengths of observed $\mathrm{He}$ II lines. (Note: of all the lines, He II $\lambda 1640$ provided the strictest constraints on the continuum shape and covering fraction in Korista et al. 1997b.)

The lines that form the ratios in Figure 4 are not particularly bright. We therefore utilized, for analysis, the high signal-to-noise, high-resolution spectra of a small sample of quasars obtained by Baldwin et al. (1996). Many of the lines required are at the limit of detection despite the high quality of these observations. Other lines are definitely detected but have large estimated errors. This translates into large uncertainties in the ratio of any two lines. As a result, attempts to detect the presence or degree of microturbulence on the basis of these observations will have large uncertainties.

Figure 5 shows the range of inferred $V_{\text {turb }}$ from line ratios for various objects in Baldwin et al. (1996). We first note a few items omitted from the figure. The ratio $\mathrm{N}$ III 990/N III] 1750 is completely uncertain for every quasar in Baldwin et al. (1996). This ratio is therefore omitted from the figure even though it could be a powerful tool. In addition the quasars or quasar components identified as Q1451, Q1623(A), and Q0207(B) are also completely uncertain in every ratio and are also omitted from the figure. Of the remaining quasars or quasar components, Figure 5 suggests that these objects typically have microturbulent velocities of $V_{\text {turb }} \sim 10^{3} \mathrm{~km} \mathrm{~s}^{-1}$.

Unfortunately, the large uncertainty in the line ratios in many of these objects prevents thermal broadening from being ruled out. However the spectra of two objects, H0335 and Q03408, are accurate enough to deduce the presence of turbulence and rule out thermal broadening. The line ratio Fe II 1787/Si II 1808 of quasar H0335 has an LOC inferred turbulence of $2.7<\log \left(V_{\text {turb }}\right)<3.9$ while the same ratio implies microturbulent velocities greater than $10 \mathrm{~km} \mathrm{~s}^{-1}$ in the quasar Q03408. In addition, the Si II 1305/ Si II 1808 ratio of Q03408 suggests a microturbulent velocity greater than $10^{2} \mathrm{~km} \mathrm{~s}^{-1}$. Microturbulence may explain the problems encountered with the strength of Si II and Fe II $\lambda 1787$ (UV191) in quasar spectra.

In addition to the individual quasars of Ferland et al. (1996) we attempted to determine the presence of microturbulence in a mean quasar sample (Zheng et al. 1997) and found mixed results. Of the six microturbulence sensitive indicators identified in Figure 4, three of them could not be calculated because these involve Si II $\lambda 1808$ and this line is not present in the list of composite quasar emission-line strengths. Of the remaining three, the ratios C II $\lambda 1335 / \mathrm{C}$ II] $\lambda 2326$ and C III $\lambda 977 / \mathrm{C}$ III] $\lambda 1909$ give $V_{\text {turb }}$ values off the low end of the velocity scale in Figure 4 (i.e., toward little or no microturbulence). This is true regardless of whether the whole sample was used or just its radio-loud or radio-quiet subsets.

On the other hand the $\mathrm{N}$ III $\lambda 990 / \mathrm{N}$ III] $\lambda 1750$ ratio (which could not be obtained for the quasars studied by 


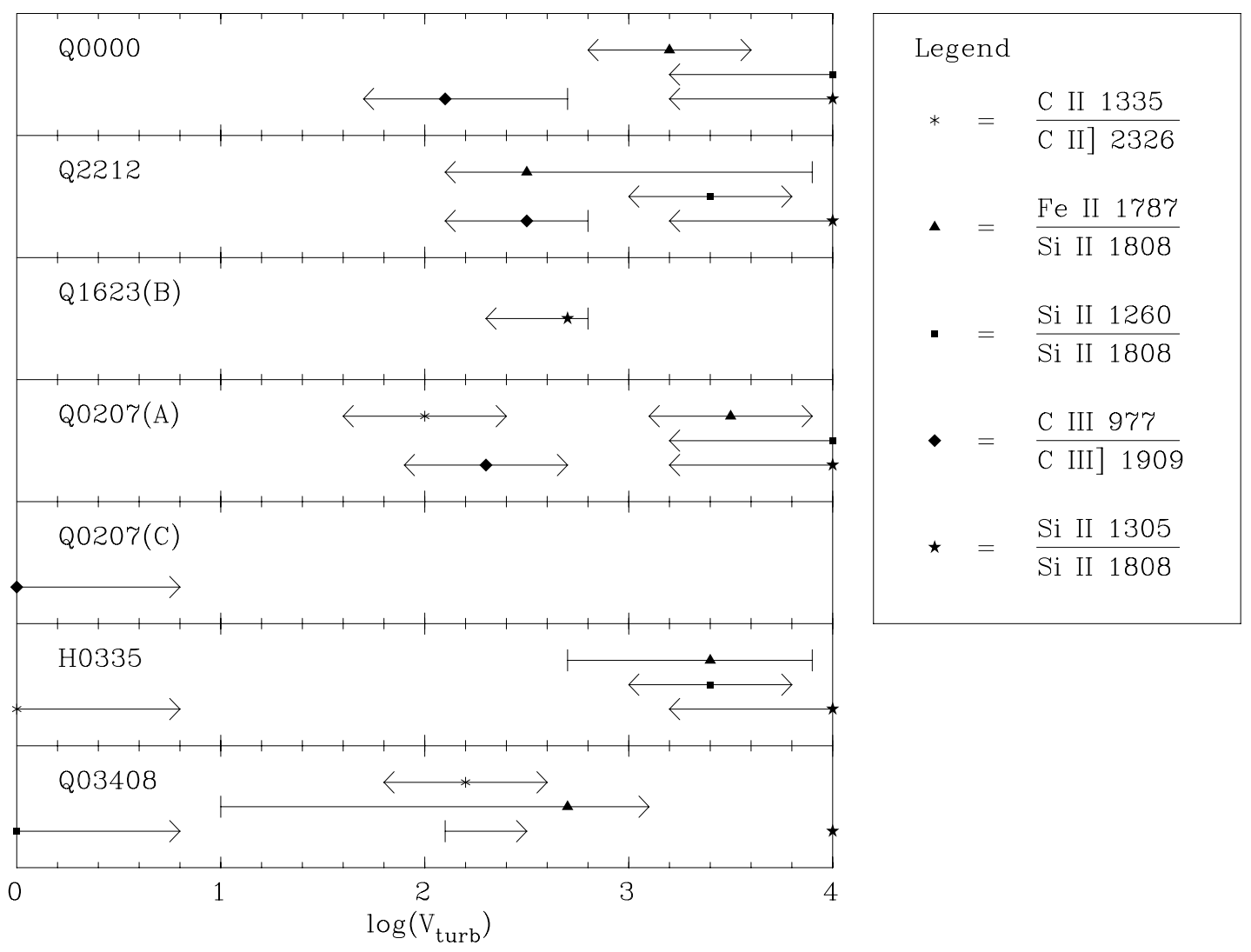

FIG. 5. - Line ratio microturbulent velocities for selected quasars in Ferland et al. (1996) as inferred by the standard LOC model

Baldwin et al. 1996) yield $\log \left(V_{\text {turb }}\right)<2.2$ for radio-loud objects and $\log \left(V_{\text {turb }}\right) \approx 2.6-3.6$ for radio-quiet objects $\left[\log \left(V_{\text {turb }}\right) \approx 1.9-2.9\right.$ for the combined sets $]$. This disparate result between the radio-loud and radio-quiet objects suggests that a study comparing turbulence sensitive indicators for radio-loud and radio-quiet quasars is worth pursuing.

The results consistent with microturbulence for the small number of individual quasars in Baldwin et al. (1996) contradicts the C II $\lambda 1335 / \mathrm{C}$ II] $\lambda 2326$ and $\mathrm{C}$ III $\lambda 977 / \mathrm{C}$ III] $\lambda 1909$ line ratios of the composite quasar spectra. Possible explanations of these contradictory results are discussed at the end of the next section.

\section{CONCLUSIONS AND FUTURE DIRECTIONS}

In this paper we have investigated the influence of largescale microturbulence on broad emission lines in AGNs. As a result of comparing models with observations we make the following conclusions:

1. Microturbulence does strongly affect the spectrum of a photoionized cloud and may be needed to explain the observed smooth profiles. Its effects, however, have received very little previous study. The existence of microturbulence is fundamental since it would constrain cloud scenarios. The static three-phase interstellar medium, for instance, would have thermal motions alone. Magnetically confined clouds can have a very large microturbulence.

2. Observations do not rule out the presence of microturbulence in most cases. In a few cases a microturbulent velocity of the order of $10^{3} \mathrm{~km} \mathrm{~s}^{-1}$ is needed to reproduce sensitive line ratios.

3. Intensities of bright quasar emission lines tend to increase as turbulent velocities increase. This may provide part of the solution to the fact that observed equivalent widths of bright quasar lines are larger than expected assuming only thermal broadening and photoionization by the observed continuum.

4. The equivalent width of $L y \alpha$ increases by a factor of $\sim 4.6$ over the range of microturbulent velocities we consider, but most other bright emission lines do not increase as much. The result is that the ratio of bright lines relative to $\mathrm{Ly} \alpha$ decreases with increasing microturbulent velocity. The decrease is by less than a factor of 10 , keeping most of the line ratios within a factor of a few of observational constraints. A disparity of this size may be corrected by altering the incident continuum shape, since this determines the ratio of cooling lines relative to Ly $\alpha$ (Ferland 1999). Such fine-tuning, however, is not the focus of this work.

5 . The observed large $\mathrm{H} \beta / \mathrm{Ly} \alpha$ ratio, long a problem for theoretical models, is made worse with increased microturbulent velocities. This could be a deficiency in the radiative transport methods (Netzer et al. 1995) or a serious problem.

6. When a range of microturbulent velocities is present, a high level of microturbulence may be difficult to detect. Nondetection of Al II $\lambda 1671$ and a weak detection of Al III $\lambda 1859$ therefore does not necessarily imply low $\left(<10^{3} \mathrm{~km}\right.$ $\mathrm{s}^{-1}$ ) microturbulence. Unfortunately, a range of microturbulent velocities, by comparison to models with constant high-velocity microturbulence, produces less He II $\lambda 1640$ emission relative to Ly $\alpha$. The He II energy budget problem therefore remains unresolved.

7. The line spectrum becomes brighter for larger values of microturbulence, requiring the cloud covering factor to be smaller than in thermal line width models. 
8. The amounts of microturbulence inferred from different line ratios in the composite quasar spectrum are contradictory, while some line ratios in the sharp lined quasars of Baldwin et al. (1996) require turbulence. Are these two samples indeed different? Can quasars be classified by the level of microturbulence?

9. The main result of this study has been the identification of emission-line ratios that could serve as diagnostics of microturbulence (or extrathermal local line widths). High signal-to-noise observations of crucial trans-Ly $\alpha$ lines, in individual objects, would be essential in clarifying the physical nature of the emitting clouds.

Research in nebular astrophysics at the University of Kentucky is supported by the NSF and NASA. We thank the referee for a careful reading of the manuscript.
Alexander, T., \& Netzer, H. 1994, MNRAS, 270, 781

Arav, N., Barlow, T. A., Laor A., \& Blandford, R. D. 1997, MNRAS, 288, 1015

Arav, N., Barlow, T. A., Laor, A., Sargent, W. L. W, \& Blandford, R. D. 1998, MNRAS, 297, 990

Baldwin J. A. 1997, in ASP Conf. Ser. 113, in Emission Lines in Active Galaxies: New Methods and Techniques, IAU Colloq. 159, ed. B. M Peterson, F.-Z. Cheng, \& A. S. Wilson (San Francisco: ASP), 81, 1997

Baldwin, J. A., Ferland, G. J., Korista, K. T., Carswell, R. F., Hammann, F., Phillips, M. M., Verner, D., Wilkes, B. J., \& Williams, R. E. 1996, ApJ, 461, 644

Baldwin, J. A., Ferland, G. J., Korista, K. T., \& Verner, D. 1995, ApJ, 455, 119

Bottorff, M. C., \& Ferland, G. J. 2000, MNRAS, 316, 103

Brotherton, M. S., Wills, B. J., Francis, P. J., \& Steidel, C. C. 1994, ApJ, 430, 495

Davidson, K., \& Netzer, H. 1979, Rev. Mod. Phys., 51, 715

Dietrich, M., Wagner, S. J., Courvoiser, T. J.-L., Bock, H., \& North, P. 1999, A\&A, 351, 31

Emmering, R. T., Blandford, R. D., \& Shlosman, I. 1992, ApJ, 385, 460

Ferland, G. J. 1992, ApJ, 389, L63

. 1999, in ASP Conf. Ser. 162, in Quasars and Cosmology, ed.

G. Ferland \& J. Baldwin (San Francisco), 147

\section{EFERENCES}

Ferland, G. J. 1999, Rev. Mexicana Astron. Astrofis. Ser. de Conf., 9, 153

Ferland, G. J., Baldwin, J. A., Korista, K. T., Hammann, F. Carswell, R. F., Phillips, M., Wilkes, B., \& Williams, R. E. 1996, ApJ, 461, 683

Frank, J., King, A. R., \& Raine, D. J. 1992, Accretion Power in Astrophysics (2d ed.; Cambridge: Cambridge Univ. Press)

Hamann, F., Choen, R. D., Shields, J. C., Burbidge, E. M., Junkkarinen, V., \& Crenshaw, D. M. 1998, ApJ, 496, 761

Hamann, F., Ferland, G. J. 1999, ARAA, 37, 487

Korista, K. T., Baldwin, J. A., \& Ferland, G. J. 1998, ApJ, 507, 24

Korista, K. T., Baldwin, J., Ferland, G., \& Verner, D. 1997a, ApJS, 108, 401

Korista, K. T., Ferland, G., \& Baldwin, J. 1997b, ApJ, 487, 555

Korista, K. T., \& Goad, M. R. 2000, ApJ, 536, 284

Murray, N., \& Chiang, J. 1998, ApJ, 494, 125

Netzer, H., Brotherton, M. S., Wills, B. J., Han, M., Wills, D., Baldwin, J. A., Ferland, G. J., \& Brown, I. W. A. 1995, ApJ, 448, 27

Peterson, B. M. 1993, PASP, 105, 247

Rees, M. J. 1987, MNRAS, 228, 47

Shields, J. C., Ferland G. J., \& Peterson, B. J. 1995, ApJ, 441, 507

Verner, E. M., Verner, D. A., Korista, K. T., Ferguson, J. W., Hamann, F. \& Ferland, G. J. 1999, ApJS, 120, 101

Zheng, W., Kriss, G. A., Telfer, R. C., Grimes, J. P., \& Davidsen, A. F. 1997, ApJ, 475, 469 